\title{
Consumo di alcol e tabacco negli studenti infermieri: uno studio osservazionale
}

\author{
Francesco Galli ${ }^{\circ}$ Fabiana Piergigli ${ }^{\S}$, Carla Pierini*, Stefano Fornaroli^, \\ Martina Cocchiglia**, Dania Comparcinis\&
}

\section{Introduzione}

L'Unione Europea è nel mondo la regione con la più elevata proporzione di consumatori di bevande alcoliche e con i più alti livelli di consumo (Anderson et al., 2005).

L'alcol è, in ordine di rilevanza, il terzo fattore di rischio per malattia e morte prematura dopo il fumo e l'ipertensione, più rilevante dell'ipercolesterolemia e del sovrappeso, tre volte più importante del diabete e cinque volte più dell'asma (WHO, 2002).

Oltre ad essere una sostanza in grado di indurre dipendenza ed essere la causa di circa 60 differenti condizioni di malattia e infortunio, l'alcol è responsabile di danni sociali, mentali ed emotivi, compresi la criminalità e le violenze in ambito familiare, che ogni anno costano all'Unione Europea circa 124 miliardi di euro di oneri tangibili (Baumberg \& Anderson, 2005).

L'alcol, spesso, viene assunto proprio per i suoi effetti tossici, e molti bevitori, in particolar modo i giovani, assumono l'alcol deliberatamente e consapevolmente per esserne intossicati, ovvero per ubriacarsi; tuttavia, questa modalità di bere rappresenta spesso la causa di molti danni sociali. Inoltre, il rischio degli effetti sociali negativi dell'alcol più comunemente conosciuti, come l'essere coinvolti in una rissa, danneggiare la vita familiare, il matrimonio, il lavoro, gli studi, le amicizie o la vita sociale, aumenta in proporzione alla quantità di alcol assunta, senza alcun effetto soglia evidente.

L'aumento del rischio ai livelli più bassi di consumo è in gran parte dovuto a bevitori di piccole quantità che occasionalmente assumono quantità maggiori d'alcol (Rem \& Gmel, 1999).

Negli ultimi anni in Italia si è assistito ad un cambiamento nei consumi di alcol per lo più dovuti alla cultura del tempo libero. Il cambiamento nel modello di consumo ha visto una diminuzione del consumo quotidiano, tipico del tradizionale modello

- Infermiere. Dipartimento Dipendenze Patologiche Asur Marche, Area Vasta 2, Ancona.

$\S$ Sociologo. A.T.I. con Dipartimento Dipendenze Patologiche Asur Marche, Area Vasta 2, Ancona.

* Dirigente Medico. Dipartimento Dipendenze Patologiche Asur Marche, Area Vasta 2, Ancona.

^Dirigente Sociologo. Dipartimento Dipendenze Patologiche Asur Marche, Area Vasta 2, Ancona.

** Studente Corso di Laurea Magistrale in Scienze Infermieristiche ed Ostetriche, Università Politecnica delle Marche, Ancona.

§§ Infermiera, Dottore di Ricerca Università G. d'Annunzio, Chieti. Dipartimento Gastroenterologico e dei Trapianti, Azienda Ospedaliero Universitaria Ospedali Riuniti "Umberto I - G.M. Lancisi - G. Salesi", Ancona. mediterraneo, un aumento di chi beve fuori dai pasti e occasionalmente, con un adeguamento al modello di consumo nord europeo.

Questo comportamento è più pronunciato tra gli adulti fino a 44 anni e tra i giovani di età compresa tra i 18 e i 24 anni, nei quali è forte la crescita del consumo di alcol fuori pasto.

Tutti i sistemi di reportistica nazionale concordano sul fatto che i giovani di 18-24 anni sono una delle categorie in cui è più diffuso il consumo a maggior rischio, che comprende il consumo fuori pasto, il consumo abituale elevato e il binge drinking, una modalità di consumo di quattro/cinque o più Unità Alcoliche (UA), che provoca un'alcolemia superiore a $0,8 \mathrm{~g} / \mathrm{l}$, in circa 2 ore.

Allo stesso modo della dipendenza da alcol, il tabagismo è considerato una dipendenza patologica in quanto l'uso di tabacco determina una modificazione definitiva del cervello che rende molto difficile l'astinenza e, nel caso di cessazioni, si associa ad un'elevata probabilità di recidive (Leshner, 1997). I risultati di un recente studio svolto in Italia (Gallus et al., 2011) hanno evidenziato che nel 2010 la prevalenza complessiva del fumo era pari al $21,7 \%$, rispettivamente il $23,9 \%$ tra gli uomini e il $19,7 \%$ tra le donne, con un consumo medio giornaliero di tredici sigarette; tuttavia il $38 \%$ dei fumatori consumava più di un pacchetto al giorno; i soggetti con dipendenza da nicotina di grado moderato-grave, rilevato attraverso l'utilizzo del test di Fagerström (Heatherton et al., 1991), erano circa il $20 \%$ dei fumatori che, anche quando motivati a smettere di fumare, non riuscivano a farlo senza un supporto esterno.

Il fumo di tabacco in Italia resta la principale causa di mortalità prevenibile; le stime dell'ISTAT del 2012 attribuiscono al fumo di tabacco dalle 70.000 alle 83.000 morti l'anno, circa il $12,5 \%$ della mortalità totale e oltre una su quattro di queste morti riguarda soggetti di età compresa tra i 35 e i 65 anni.

Secondo i dati raccolti nel 2012 dal sistema di sorveglianza PASSI il 27,9\% degli italiani adulti nella fascia 18-69 anni è classificabile come fumatore, mentre il $54 \%$ è classificato come non fumatore e il $18,2 \%$ come ex fumatore.

La fascia di età 25-35 anni è quella a maggiore prevalenza (33\%), seguita da quella 18-24 (32\%) e 35-49 anni (29\%).

Il fumo è dannoso ad ogni età, tuttavia è stato dimostrato che il rischio di contrarre una patologia a esso correlata (cardiovascolare, oncologica, pneumologica) è strettamente dipendente dall'età di inizio: un individuo che inizia a fumare a 15 anni ha una probabilità tre volte superiore di ammalarsi di tumore rispetto a uno che inizia a fumare a 20 anni (Simonato et al., 2001; McCarron et al., 2001).

La fascia di popolazione che maggiormente detiene abitudini rischiose per la salute è rappresentata dai giovani adolescenti, sia perché percepiscono in misura minore la possibilità del rischio sia perché spesso, adottando comportamenti sbagliati, 
si sentono omologati al gruppo e quindi maggiormente accettati.

Lo studente universitario rappresenta una fascia d'età compresa generalmente tra i 18 e i 24 anni, definita giovane adulto, in quanto, non essendo più un adolescente ma neanche un adulto ancora completamente formato, presenta alcune caratteristiche tipiche dell'adolescente e altre invece dell'adulto.

I risultati di alcuni studi presenti in letteratura (Reeve et al., 2013; Deasy et al., 2016) hanno evidenziato che tra gli studenti universitari, gli studenti infermieri vivono esperienze ad elevato rischio di stress psico-emotivo poiché sperimentano un ambiente di apprendimento complesso caratterizzato da una componente accademica che una componente clinica (Wolf et al., 2015).

Il perpetuarsi di esperienze stressanti, infatti, potrebbe aumentare il rischio di sviluppare dipendenze patologiche all'interno di una popolazione di studenti che rappresentano gli infermieri del futuro, categoria professionale particolarmente sensibilizzata verso l'adozione e il mantenimento di stili di vita sani.

Pertanto, è possibile ipotizzare che anche la presenza di dipendenze da fumo e soprattutto da alcol potrebbero influenzare negativamente l'esperienza di apprendimento e il successo accademico degli studenti infermieri, inficiando lo sviluppo delle competenze che gli stessi sono chiamati ad acquisire in ambito universitario per poter essere inseriti nel futuro mondo professionale.

Inoltre, anche la capacità degli studenti di garantire sicurezza e qualità assistenziale durante il tirocinio clinico e nei futuri luoghi di lavoro potrebbe essere compromessa, considerando le numerose comorbidità associate a tali dipendenze.

A livello internazionale sono stati condotti numerosi studi per valutare la dipendenza da alcol e tabacco tra gli studenti infermieri (López-Maldonado, 2011) e la sola dipendenza da alcol (Watson et al., 2006; Burke \& McCarthy, 2011; Sotos et al., 2015) o da tabacco (Chalmers et al., 2002; Patkar et al., 2003; Suzuki et al., 2005; Molina et al., 2010) dai quali è emerso che il comportamento degli studenti rispetto alle abitudini alcoliche e tabagiche non è omogeneo nelle varie parti del mondo.

In Italia, ad oggi, pochi studi hanno esplorato tali tematiche nel contesto universitario infermieristico (Quattrin et al., 2010; Biraghi \& Tortorano, 2010) e i dati raccolti dagli autori si riferiscono a popolazioni di studenti afferenti a sole Regioni del Nord Italia (Lombardia e Friuli Venezia Giulia).

\section{Obiettivo dello studio}

L'obiettivo dello studio è quello di determinare il consumo rischioso e dannoso di alcol e di quantificare il livello di dipendenza da tabacco in una popolazione di studenti infermieri italiani afferenti ad un Corso di Laurea del Centro Italia.

\section{Materiali e metodi}

\section{Disegno dello studio}

Studio osservazionale, trasversale, monocentrico.

\section{Descrizione del questionario}

Il questionario utilizzato per la raccolta dati si compone di tre sezioni: la prima raccoglie le informazioni socio-demografiche dei partecipanti (età, genere, anno di corso di studio, specificando se in corso o fuori corso, esperienze lavorative pregresse o in corso, stato civile, specificando se con figli a carico, uso di tabacco e uso di alcol, specificando se attuale o pregresso, a che età il primo contatto e se anche i familiari e/o amici ne fanno uso); la seconda sezione, finalizzata alla raccolta dati relativi al consumo di alcol da parte dei partecipanti, prevede la compilazione del test "Alcohol Use Disorders Identification Test" (AUDIT) (Babor et al., 2001), elaborato dall'Organizzazione Mondiale della Sanita per l'identificazione del consumo problematico di alcol; nello specifico, il test è costituito da 10 domande che esplorano le modalità del consumo di alcol; ogni item viene calcolato considerando un punteggio che varia da 0 a 4, ad eccezione degli ultimi due item per i quali si considera un punteggio che varia da 0 a 2 , secondo uno schema di interpretazione prestabilito: punteggi compresi tra 0-8 indicano l'assenza di un consumo rischioso di alcol, punteggi tra 914 indicano un consumo a rischio oppure che il soggetto ha o ha avuto problemi alcol-correlati (infortuni o forti bevute occasionali) ma, probabilmente, non si tratta di una persona fisicamente alcol-dipendente, invece, un punteggio pari o maggiore di 15 evidenzia problemi alcol-correlati e/o si tratta di un soggetto alcol-dipendente.

La terza sezione del questionario comprende la versione italiana del test "Fagerström Test for Nicotine Dependance" (FTND) (Heatherton et al., 1991) finalizzato alla raccolta dati relativi al grado di nicotino-dipendenza del soggetto.

Nello specifico, il test FTND è costituito da 6 domande che esplorano le modalità del consumo di tabacco e fanno riferimento alla quantità di sigarette fumate durante la giornata, quando vengono fumate e alla impossibilità di non fumare dove vietato; ogni item viene calcolato considerando un punteggio che varia da 0 a 1 , ad eccezione degli item 1 e 4 per i quali si considera un punteggio che varia da 0 a 4 , secondo uno schema di interpretazione prestabilito: punteggi compresi tra $0-2$ indicano un grado di dipendenza molto basso, punteggi tra 3-4 un grado di dipendenza basso, tra 5-6 un grado di dipendenza medio, tra 7-8 un grado di dipendenza elevato, mentre punteggi tra 9-10 indicano un grado di dipendenza molto elevato.

\section{Popolazione, setting e procedure di studio}

Lo studio è stato condotto tra Ottobre e Dicembre 2015, in un campione di convenienza di studenti afferenti al Corso di Laurea in Infermieristica di Ancona $(n=364)$ Facoltà di Medicina e Chirurgia dell'Università Politecnica delle Marche attraverso l'utilizzo di un questionario auto-somministrato accompagnato da una lettera di presentazione nella quale sono state specificate le indicazioni per la compilazione, le finalità dello studio e la modalità di analisi dei dati che avrebbe garantito l'anonimato dei partecipanti.

Tutti coloro che accettavano volontariamente di partecipare allo studio sono stati invitati a compilare la prima sezione del questionario, relativa ai dati socio-demografici, nella quale si dichiara anche l'eventuale consumo di alcol e/o di tabacco. 
Coloro che affermavano di consumare alcol venivano invitati a compilare il test AUDIT (Babor et al., 2001) e coloro che dichiaravano di consumare tabacco venivano invitati a compilare anche la scala FTND (Heatherton et al., 1991).

\section{Criteri di inclusione ed esclusione}

Sono stati reclutati tutti gli studenti regolarmente iscritti al primo, secondo e terzo anno del Corso di Laurea in Infermieristica e sono stati inclusi tutti coloro che accettavano volontariamente di partecipare allo studio.

Sono stati esclusi tutti gli studenti fuori corso e gli studenti assenti il giorno concordato per la somministrazione dei questionari.

\section{Analisi dei dati}

L'elaborazione statistica è stata effettuata mediante programma statistico EPINFO 3.5.1 per Windows.

Le variabili continue sono state sintetizzate mediante media e deviazione standard (DS) e le variabili categoriche mediante frequenza e percentuale.

Le variabili dipendenti (punteggi test AUDIT e FTND) e le variabili indipendenti (età, sesso e anno di corso) sono state messe in relazione per misurare la forza dell'associazione tramite coefficiente di regressione lineare di test di Fisher, Rischio Relativo e Odds Ratio.

Sono stati considerati come significativi i valori di $\mathrm{p}<0.005$.

\section{Risultati}

\section{Caratteristiche socio-demografiche del campione}

Hanno partecipato allo studio 320 studenti su 364 contattati (tasso di risposta: 87.9\%).

In base all'anno di Corso frequentato dai partecipanti, si distinguono: 96 (30\%) studenti del I anno, 109 (34.1\%) del II anno e 115 (35.9\%) del III anno.

La maggioranza del campione è costituita dal genere femminile $(\mathrm{n}=241,75.3 \%)$ e l'età media è pari a 21.6 anni $(\mathrm{DS}=3.8)$. Circa la metà del campione $(\mathrm{n}=161,50.3 \%)$ non ha esperienza lavorativa, né in corso né pregressa, 30 (9.4\%) studenti, invece, hanno dichiarano di svolgere un'attività lavorativa durante il corso di studio.

Per quanto riguarda lo stato civile, la quasi totalità del campione $(\mathrm{n}=300,93.8 \%)$ è nubile o celibe, $20(6.2 \%)$ studenti sono sposati o conviventi e $12(3.8 \%)$ sono genitori.

Tra i dati socio-demografici è stata esplorata l'eventuale presenza di abitudini alcoliche e tabagiche, sia dei soggetti esaminati sia dei loro familiari e l'età del primo contatto con la sostanza.

L'82.8\% (n = 265) degli studenti ha affermato di far uso di alcol e il $35.9 \%(n=115)$ di far uso di tabacco.

Tra questi $154(57.2 \%)$ consumano soltanto bevande alcoliche, $4(1.5 \%)$ soltanto tabacco e $111(34.6 \%)$ entrambe le sostanze, il $15.9 \%(n=51)$ dichiara di non bere e di non fumare.
Le altre caratteristiche demografiche dei partecipanti sono riportate in Tabella 1.

Tab. 1 - Caratteristiche del campione $(n=320)$

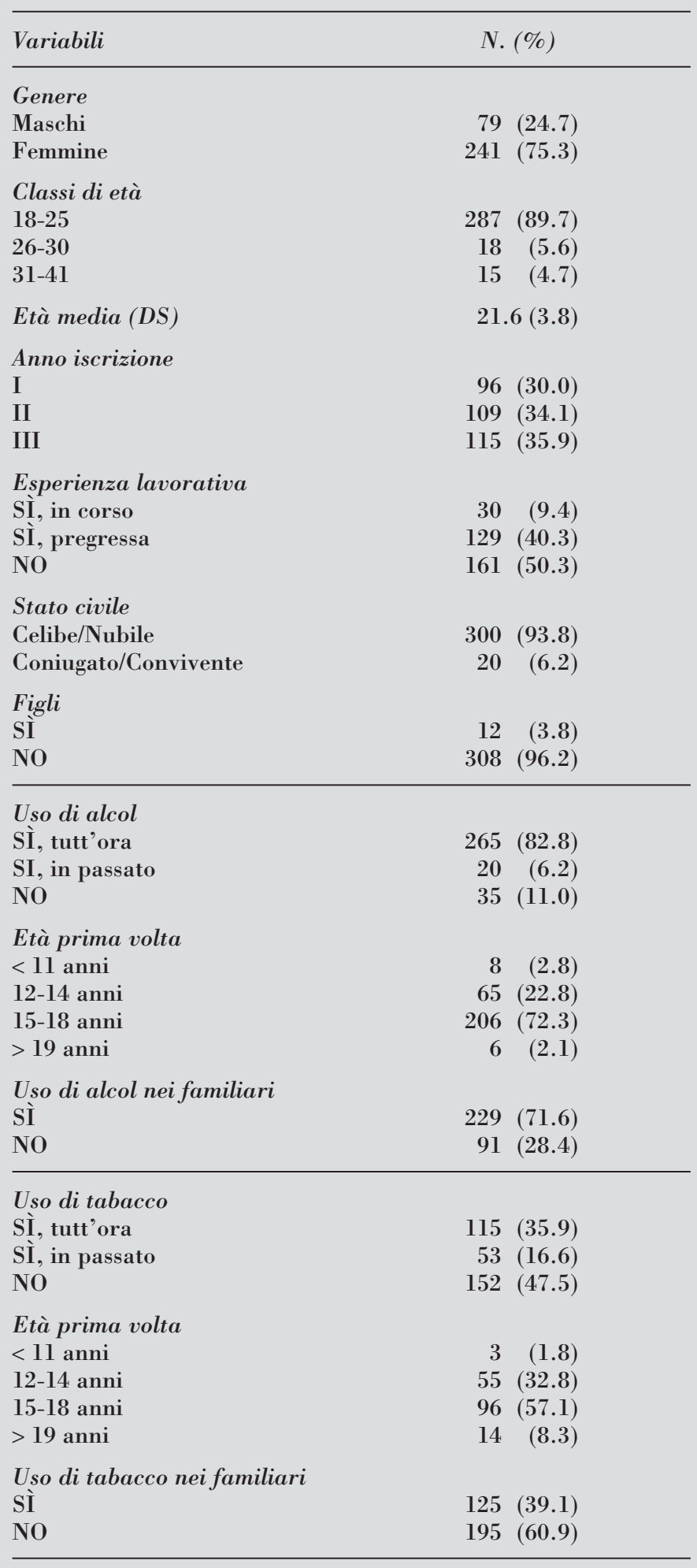

\section{Consumo di alcol (test AUDIT)}

Su un campione di studenti che hanno dichiarato di usare alcolici $(\mathrm{n}=265$, di cui 71 maschi e 194 femmine), il $31.3 \%$ 
beve almeno una bevanda alcolica al mese, quasi la metà $(43.8 \%)$ ogni settimana, il $17.7 \%$ fino a 3 volte a settimana e il $6.8 \%$ fino a 4 o più volte a settimana.

In una giornata tipica quando bevono, la maggioranza del campione $(72.8 \%)$ ha dichiarato di prendere 1 o 2 bevande che contengono alcol, invece 19 studenti $(7.2 \%)$ fino a 10 o più bevande alcoliche; tuttavia il $72.5 \%$ non beve mai 6 o più bevande alcoliche in un' unica occasione.

Il $18.9 \%$ del campione dichiara di avere questa tipologia di consumo meno di una volta al mese, il $2.3 \%$ con una frequenza mensile, il $4.5 \%$ settimanale e l'1.9\% ogni giorno o quasi. Suddividendo i soggetti in base al punteggio ottenuto al test AUDIT sono stati evidenziati 3 gruppi: il primo con AUDIT compreso tra 0-8 $(\mathrm{n}=238$, di cui 57 maschi e 181 femmine) rappresenta la maggioranza del campione $(89.8 \%)$ che non presenta un consumo rischioso di alcol, il secondo gruppo con AUDIT compreso tra 9-14 $(\mathrm{n}=21$, di cui 8 maschi e 13 femmine) rappresenta il $7.9 \%$ del campione che è a rischio di problemi correlati al consumo di bevande alcoliche e, infine, il terzo gruppo con AUDIT $\geq 15(n=6$, tutti di sesso maschile) rappresenta il $2.3 \%$ del campione che risulta avere problemi alcol-correlati o di dipendenza dalla sostanza.

Dividendo i soggetti per la variabile "genere" è emerso che il 93.3\% delle donne non ha un consumo rischioso di alcol, il $6.7 \%$ ha un consumo rischioso e nessun soggetto di genere femminile ha problemi alcol-correlati.

Per quanto riguarda il genere maschile, invece, $\mathbf{l}^{\prime} \mathbf{8 0 . 3 \%}$ non presenta un consumo rischioso di alcolici, l'11.3\% presenta un consumo a rischio e l'8.5\% risulta avere problemi di dipendenza da alcol.

La differenza dei punteggi assegnati al test AUDIT tra i due sessi è statisticamente significativa $(p<0.005)$.

\section{Consumo di tabacco (test di Fagerström)}

Su un campione di studenti che hanno dichiarato di usare tabacco ( $\mathrm{n}=115$, di cui 33 maschi e 82 femmine), la maggioranza $(72.2 \%)$ fuma meno di 10 sigarette al giorno, il $22.6 \%$ meno di 20 sigarette al giorno e soltanto 6 studenti (5.2\%) consumano fino a 30 sigarette in una giornata.

Tra coloro che fumano il $58.3 \%$ accende la prima sigaretta entro 5 minuti dopo il risveglio, il $32.1 \%$ entro la prima ora e il $9.6 \%$ dopo un'ora. 63 studenti fumatori (54.8\%) hanno dichiarato di fumare anche se sono malati.

Suddividendo i soggetti in base al punteggio ottenuto al test di Fagerström, più della metà $(61.7 \%)$ presenta un grado di dipendenza dalla nicotina molto basso, il $18.3 \%$ dei soggetti ha un grado di dipendenza basso, il $13.9 \%$ del campione risulta avere una moderata dipendenza dalla nicotina, invece, il 5.2\% presenta un grado di dipendenza alto e lo $0.9 \%$ molto alto.

Per meglio identificare i soggetti con problemi di dipendenza dalla nicotina dai soggetti sani, il campione di fumatori è stato suddiviso in due gruppi in base al punteggio totale ottenuto al test (FTND): il primo gruppo con FTND compreso tra 0-4 (n = 92, di cui 23 maschi e 69 femmine) che presenta una bassa dipendenza e il secondo gruppo con FTND $\geq 5(n=23$, di cui 10 maschi e 13 femmine) con elevata dipendenza tale da richiedere una terapia sostitutiva.

Stratificando i partecipanti per il genere, si evidenzia che tra le femmine viene riscontrata una elevata dipendenza dalla nicotina in 3 soggetti su $82(3.6 \%)$, rispetto al $84.1 \%$ che risulta avere una bassa dipendenza.

Per quanto riguarda il genere maschile, invece, una dipendenza alta, tale da richiedere una terapia sostitutiva, si osserva in 4 soggetti su $33(12.1 \%)$, tuttavia non si evidenzia una differenza statisticamente significativa tra i punteggi assegnati al test FTND in relazione al genere $(p=0.08)$.

\section{Analisi di correlazione}

Per valutare l'influenza delle variabili socio-demografiche e dei punteggi assegnati ai test AUDIT e FTND sul rischio di sviluppare una dipendenza da alcol o da tabacco, è stato utilizzato un modello di regressione lineare, inserendo come variabili dipendenti i punteggi AUDIT e FTND e come variabili indipendenti l'età, il genere e l'anno di corso.

Nella Tabella 2 sono rappresentati i dati di correlazione tra i punteggi attribuiti ai test AUDIT e FTND e le variabili indipendenti prese in considerazione.

Tab. 2 - Modello di regressione tra $i$ punteggi attribuiti $i$ test AUDIT e FTND e le variabili sociodemografiche

\section{Punteggi Test AUDIT*}

Variabili

$\begin{gathered}\text { Coeffi- Errore } \\ \text { ciente Standard }\end{gathered}$
Standue

\begin{tabular}{lrrrrr}
\hline Età & -0.386 & 0.591 & 0.427 & 0.514 & 0.00 \\
Genere & -0.273 & 0.067 & 16.366 & 0.000 & 0.06 \\
Anno di Corso & -0.131 & 0.129 & 1.031 & 0.310 & 0.00 \\
\hline
\end{tabular}

Punteggi Test FTND*

\begin{tabular}{|c|c|c|c|c|c|}
\hline Variabili & $\begin{array}{l}\text { Coeffi- } \\
\text { ciente }\end{array}$ & $\begin{array}{c}\text { Errore } \\
\text { Standard }\end{array}$ & $F$ & p-value & $r^{2}$ \\
\hline Età & -3.261 & 1.003 & 10.573 & 0.001 & 0.09 \\
\hline Genere & 0.185 & 0.105 & 3.100 & 0.081 & 0.03 \\
\hline Anno di Corso & -0.098 & 0.198 & 0.244 & 0.622 & 0.00 \\
\hline
\end{tabular}

* Variabile dipendente.

Se si prendono in esame coloro che fumano in relazione a quanti usano alcolici, si osserva che l'associazione causa effetto è pari a 7.15, mentre l'OR è di $9.18\left(\mathrm{~F}=25.439, \mathrm{r}^{2}=0.07\right.$, $\mathrm{p}=\mathbf{0 . 0 0 0 0 0 1 )}$. Nella Tabella 3 sono riportate le correlazioni tra il consumo di alcol e di tabacco negli studenti e il relativo consumo nei loro familiari.

Tab. 3 - Analisi di correlazione del consumo di alcol e tabacco tra gli studenti e i loro familiari

Consumo alcol/tabacco negli studenti*

\begin{tabular}{lccccc} 
Variabili & $R R$ & OR & $95 \%$ CI & p-value & $r^{2}$ \\
\hline $\begin{array}{l}\text { Consumo di alcol } \\
\text { nei familiari }\end{array}$ & 3.50 & 4.85 & $2.64-8.93$ & 0.000 & 0.09 \\
$\begin{array}{l}\text { Consumo di tabacco } \\
\text { nei familiari }\end{array}$ & 1.15 & 1.49 & $0.93-2.37$ & 0.091 & 0.01 \\
\hline
\end{tabular}

* Variabile dipendente. 


\section{Discussione}

Lo studio ha voluto indagare la prevalenza del consumo rischioso e dannoso di alcol e di tabacco in una popolazione di studenti universitari italiani, ad oggi riconosciuta da diversi autori come popolazione a rischio sia per la dipendenza da alcol (Miquel et al., 2016; O'Neill et al., 2015; Campos et al., 2011; Reavley et al., 2011; English et al., 2011; Beenstock et $a l ., 2010)$ sia per la dipendenza da tabacco (Chopra et al., 2015; Alvur et al., 2014; Nasirian et al., 2013) o per entrambe le dipendenze (Gignon et al., 2015; Rueger et al., 2012).

Dai risultati dello studio è emerso che, seppur in minima parte rispetto al campione totale considerato, gli studenti infermieri manifestano problemi patologici per quanto riguarda il consumo di alcol e di tabacco, infatti il $2.3 \%$ del campione risulta avere problemi alcol-correlati o di dipendenza da alcol e il $7.1 \%$ del campione presenta un grado di dipendenza dalla nicotina alto o molto alto.

I risultati sono sovrapponibili a quelli riportati in letteratura che evidenziano percentuali simili tra gli studenti infermieri, in particolare uno studio (López-Maldonado et al., 2011) condotto a Bogotà su 237 studenti di età compresa tra 16 e 37 anni, ha evidenziato che il $2 \%$ del campione aveva problemi alcol-correlati o di dipendenza da alcol e che il $20 \%$ aveva una forte dipendenza dalla nicotina, rilevati rispettivamente con il test AUDIT e FTND. Un altro studio (Campos et al., 2011), condotto su una popolazione di quasi duemila studenti delle scuole superiori brasiliane, ha identificato che il $3.3 \%$ del campione può avere problemi di alcol-dipendenza.

Uno studio più recente su una popolazione di studenti universitari del Regno Unito ha rilevato che l'8\% del campione viene classificato come probabile alcoldipendente, sulla base del test AUDIT (O'Neill et al., 2015).

Gli strumenti utilizzati per la raccolta dati (Test AUDIT e FTND) hanno permesso, non solo di stratificare il campione in soggetti patologici e non patologici ma anche di far emergere diverse categorie che riguardano la dipendenza, sia per il consumo di alcolici, sia per la dipendenza da tabacco.

Infatti è interessante notare la presenza di un considerevole numero di studenti a rischio per entrambe le dipendenze e che rispetto ai risultati dei test somministrati rientrano nei punteggi definiti "borderline".

Nello specifico, per quanto riguarda il consumo di alcol, il $7.9 \%$ dei soggetti presenta un consumo a rischio, invece, relativamente al consumo di tabacco, il $13.9 \%$ presenta un grado di dipendenza moderato.

Pertanto i due gruppi presi in considerazione possono potenzialmente degenerare in una dipendenza patologica o molto elevata tale da richiedere terapie sostitutive, dato il consumo di queste sostanze al di sopra della norma e considerata la giovane età come una fase di cambiamento e di evoluzione.

Per quanto riguarda l'associazione dei punteggi dei test AUDIT e FTND in relazione al genere, i nostri risultati, in maniera simile a quanto riportato da altri studi effettuati su campioni di studenti universitari suggeriscono che il sesso maschile è associato ad un più alto rischio di dipendenza da alcol (Ruisoto et al., 2016; Akmatov et al., 2011; Ozgür Ilhan et al., 2008) e da tabacco (Mahfouz et al., 2014).

Tale risultato, tuttavia, deve essere interpretato considerando la maggior prevalenza dei soggetti di genere femminile all'in- terno del campione di studio e la maggiore frequenza nel campione maschile di consumo a rischio di alcol e problemi alcolcorrelati.

Per quanto riguarda invece la differenza dei punteggi assegnati al test FTND in relazione al genere, è emerso che $\mathrm{i}$ maschi, rispetto alle femmine, presentano una probabilità doppia di avere un'elevata dipendenza da tabacco $(\mathrm{RR}=$ $1.91, \mathrm{OR}=2.30$ ) .

Questo risultato potrebbe essere spiegato considerando che nell'indole maschile è presente una propensione maggiore ad assumere comportamenti a rischio e che nella fascia adolescenziale il genere femminile è riconosciuto come più maturo tendenzialmente portato ad assumere atteggiamenti responsabili (Potenza, 2006).

L'analisi di regressione lineare ha evidenziato che l'anno di corso non influenza significativamente il consumo patologico di entrambe le sostanze, invece l'età sembra avere una influenza sigificativa sui punteggi assegnati al test FTND, riguardanti la dipendenza da nicotina; nello specifico, all'aumentare dell'età si associa una maggiore dipendenza da nicotina.

Prendendo in esame coloro che fumano in relazione a quanti usano alcolici, si è osservato che l'associazione causa effetto era pari a $7.15(\mathrm{OR}=9.18, \mathrm{p}<0.005)$.

Un aspetto interessante emerso dallo studio riguarda l'associazione tra consumo di alcol o di tabacco rilevato dai test AUDIT e FTND ed il consumo delle medesime sostanze dichiarato dagli studenti infermieri nei confronti dei loro familiari.

Questo aspetto è stato esplorato in letteratura ed un recente studio ha evidenziato che l'abuso di alcol nei soggetti del nucleo familiare può essere considerato un predittore indipendente di un aumentato rischio di abuso precoce di alcol e tabacco nei giovani (McCutcheon et al., 2017).

Nel presente studio, per quanto riguarda l'uso di alcol, i soggetti consumatori di alcolici avevano 3.5 volte la possibilità di avere un familiare consumatore, mentre il rischio di diventare consumatore era quasi 5 volte più probabile nel caso di un familiare consumatore di alcolici rispetto a coloro che avevano familiari astemi.

Per quanto riguarda l'uso di tabacco, invece, l'associazione tra familiari fumatori e uso di tabacco era pari a 1.15, con un OR di 1.49.

\section{Limiti}

Il principale limite dello studio riguarda il disegno monocentrico che non consente di generalizzare i risultati all'intera popolazione di studenti infermieri italiani; tuttavia, seppur limitati ad una sola sede universitaria, i risultati dello studio hanno permesso di esplorare il fenomeno del consumo di alcolici e della dipendenza da tabacco all'interno di una campione di studenti infermieri del Corso di Laurea in Infermieristica, argomento ancora poco esplorato dalla letteratura.

Un ulteriore limite dello studio riguarda l'utilizzo di strumenti auto-somministrati che potrebbe aver inficiato la veridicità delle risposte assegnate dai partecipanti, determinando di conseguenza una sottostima o sovrastima dei fenomeni oggetti di studio. 


\section{Conclusioni}

Il consumo di bevande alcoliche e di tabacco è stato identificato come attività comune tra gli studenti infermieri, nonostante sia stata identificata una bassa percentuale di soggetti patologici per entrambe le sostanze.

Nonostante i limiti precedentemente descritti, il presente studio ha permesso di indagare una tematica ampliamente esplorata negli adolescenti e negli studenti, ma limitata nella popolazione di studenti infermieri, soprattutto nel contesto italiano.

In particolare, i risultati dello studio suggeriscono un'interessante relazione tra la presenza di consumi rischiosi e dannosi di alcol e tabacco negli studenti infermieri e il consumo delle medesime sostanze nei loro familiari (genitori, fratelli, sorelle, figli, conviventi), determinando la necessità di esplorare ulteriormente il fenomeno attraverso studi multicentrici effettuati su campioni ampi di studenti.

Recentemente i professionisti della salute hanno iniziato a riconoscere la gravità dell'abuso di sostanze alcoliche e tabacco, promuovendo campagne di prevenzione e di sensibilizzazione sulla conoscenza e consapevolezza dei rischi e dei danni connessi a tali dipendenze.

È importante, quindi, effettuare ulteriori ricerche per contribuire alla comprensione del fenomeno al fine di identificare strategie adeguate che potrebbero, soprattutto nei giovani, favorire una riduzione del consumo rischioso di alcol e di tabacco evitando l'instaurarsi di una dipendenza.

L'analisi dei fenomeni associati a queste tipologie di dipendenze tra i giovani studenti si pone, oggi, come una nuova sfida che interessa complessivamente il sistema sanitario nazionale e nello specifico i responsabili della formazione dei professionisti infermieri, riconosciuti come una delle popolazioni a rischio di sviluppare una dipendenza da alcol e/o da tabacco.

Gli studenti, considerati il futuro dell'infermieristica, dovranno occuparsi in prima persona della salute degli individui e della collettività; pertanto è importante riconoscere le problematiche legate alle dipendenze da queste tipologie di sostanze all'interno di questo gruppo di persone ed effettuare azioni preventive ed interventi precoci.

Inoltre, si rende necessario sviluppare una cultura della conoscenza e della consapevolezza in merito ai rischi e agli effetti di tali dipendenze e, contestualmente, costruire una rete di prevenzione basata sulla promozione di percorsi professionali ed umani, volti a sostenere i soggetti affetti da dipendenza da alcol e/o tabacco, attraverso programmi specifici multidisciplinari che considerino tali patologie come totalmente prevenibili e curabili.

\section{Bibliografia}

Alvur T.M., Cinar N., Oncel S., Akduran F., Dede C. (2014). Trends in Smoking among University Students between 2005-2012 in Sakarya, Turkey. Asian Pacific Journal of Cancer Prevention, 15(11): 4575-4581.

Anderson P., Baumberg B., McNeill A. (2005). Alcohol in Europe. London: Institute of Alcohol Studies.

Akmatov M.K., Mikolajczyk R.T., Meier S., Krämer A. (2011). Alcohol consumption among university students in North Rhine-
Westphalia, Germany-results from a multicenter cross-sectional study. Journal of America College Health, 59(7): 620-626.

Babor T.F., Higgins-Biddle J.C., Saunders J.B., Monteiro M.G. (2001). The Alcohol Use Disorders Identification Test Guidelines for Use in Primary Care. Geneva: World Health Organization.

Baumberg B., Anderson P. (2005). The social cost of alcohol to Europe. London: Institute of Alcohol Studies.

Beenstock J., Adams J., Beenstock M.W. (2010). The association between time perspective and alcohol consumption in university students: cross-sectional study. European Journal of Public Health, 21(4): 438-443.

Biraghi E., Tortorano A.M. (2010). Tobacco smoking habits among nursing students and the influence of family and peer smoking behavior. Journal of Advanced Nursing, 66(1): 33-39.

Burke E., McCarthy B. (2011). The lifestyle behaviours and exercise beliefs of undergraduate student nurses. A descriptive study. Health Education, 111(3): 230-246.

Campos A.J.D.B., De Castro A.J., Garcia P.P.N.S., Bosco Faria J. (2011). Consumo de álcool entre estudantes do ensino médio do município de Passos. Ciência \& Saúde Coletiva, 16(12): 47454754.

Chalmers K., Seguire M., Brown J. (2002). Tobacco use and baccalaureate nursing students: a study of their attitudes, beliefs and personal behaviours. Journal of Advanced Nursing, 40(1): 17-24.

Chopra A., Lakhanpal M., Gupta N., Suri V., Kaur G., Bhudhiraja S. (2015). The influence of occupational stress factors on nicotine dependence among students of health and nonhealth care professional colleges. Nigerian Medical Journal, 56(5): 349-352.

Deasy C., Coughlan B., Pironom J., Jourdan D., Mannix-McNamara P. (2016). Predictors of health of pre-registration nursing and midwifery students: Findings from a cross-sectional survey. Nurse Education Today, 36: 427-433.

English C., Rey J.A., Schlesselman L.S. (2011). Prevalence of hazardous alcohol use among pharmacy students at nine U.S. schools of pharmacy. Pharmacy Practice, 9(3): 162-168.

Gallus S., Muttarak R., Martinez-Sanchez J.M., Zuccaro P., Colombo P., La Vecchia C. (2011). Smoking prevalence and smoking attributable mortality in Italy, 2010. Preventive Medicine, 52(6): 434-438.

Gignon M., Havet E., Ammirati C., Traullé S., Manaouil C., Balcaen T., Loas G., Dubois G., Ganry O. (2015). Alcohol, cigarette, and illegal substance consumption among medical students: a crosssectional survey. Workplace Health \& Safety, 63(2): 54-63.

Heatherton T.F., Kozlowski L.T., Frecker R.C., Fagerström K.O. (1991). The Fagerstrom Test for Nicotine Dependence: a revision of the Fagerstrom Tolerance Questionnaire. British Journal of Addiction, 86(9): 1119-1127.

Leshner A.I. (1997). Addiction is a brain disease, and it matters. Science, 278(5335): 45-47.

López-Maldonado M.C., Luis M.A.V., Gherardi-Donato E.C.d.S. (2011). Consumo de drogas lícitas en estudiantes de enfermería de una universidad privada en Bogotá, Colombia. Revista LatinoAmericana de Enfermagem, 19(spe): 707-713.

Mahfouz M.S., Alsanosy R.M., Gaffar A.M., Makeen A. (2014). Tobacco use among university students of Jazan Region: gender differences and associated factors. BioMed Research International, 279231. doi: 10.1155/2014/279231.

McCarron P., Smith G., Okasha M., McEwen J. (2001). Smoking in adolescence and young adulthood and mortality in later life: prospective observational study. Journal of Epidemiology \& Community Health, 55(5): 334-335.

McCutcheon V.V., Agrawal A., Kuo S.I., Su J., Dick D.M., Meyers J.L. et al. (2017). Associations of parental alcohol use disorders and parental separation with offspring initiation of alcohol, cigarette and cannabis use and sexual debut in high-risk families. Addiction, doi: 10.1111/add.14003. [Epub ahead of print]. 
Miquel L., Rodamilans M., Giménez R., Cambras T., Canudas A.M., Gual A. (2016). Alcohol consumption in college students from the pharmacy faculty. Adicciones, 27(3): 190-197.

Molina A.J., Fernandez D., Delgado M., Martın V. (2010). Sensitivity and specificity of a self-administered questionnaire of tobacco use; including the Fagerstrom test. International Journal of Nursing Studies, 47(2): 181-189.

Nasirian M., Ziaaddini H., Asadollahi S. (2013). Smoking Intensity and its Relation to General Health of the Students of Kerman University of Medical Sciences, Iran. Addict Health, 5(3-4): 102107.

O'Neill G., Martin N., Birch J., Oldam A., Newbury-Birch D. (2015). The Drinkers Degree: Risk Taking Behaviours amongst Undergraduate Student Drinkers. Journal of Addiction, 965438. doi: 10.1155/2015/965438.

Ozgür Ilhan I., Yildirim F., Demirbafl H., Do an Y.B. (2008). Alcohol use prevalence and sociodemographic correlates of alcohol use in a university student sample in Turkey. Social Psychiatry and Psychiatric Epidemiology, 43(7): 575-583.

PASSI, 2011, Rapporto nazionale Passi 2011: consumo di alcol, disponibile in www.epicentro.iss.it/it/passi/rapporto2011/ ConsumoAlcol.asp.

Patkar A.A., Hill K., Batra V., Vergare M.J., Leone F.T. (2003). A comparison of smoking habits among medical and nursing students. Chest, 124(4): 1415-1420.

Potenza M.N. (2006). Should addictive disorders include nonsubstance-related conditions? Addiction, 101(1): 142-151.

Quattrin R., Zanini A., Zamolo E., Busaferro S. (2010). Are Italian nursing students healthy and having protective lifestyle behaviours? A pilot study. Annali di igiene: medicina preventive $e$ di comunità, 22(1): 83-88.

Reavley N.J., Jorm A.F., McCann T.V., Lubman D.I. (2011). Alcohol consumption in tertiary education students. BMC Public Health, 11: 545.

Reeve K.L., Shumaker C.J., Yearwood E.L., Crowell N.A., Riley J.B. (2013). Perceived stress and social support in undergraduate nursing students' educational experiences. Nurse Education Today, 33(4): 419-424.
Rehm J., Gmel G. (1999). Patterns of alcohol consumption and social consequences. Results from an 8 year follow-up study in Switzerland. Addiction, 94(6): 899-912.

Rueger S.Y., Trela C.J., Palmeri M., King A.C. (2012). SelfAdministered Web-Based Timeline Followback Procedure for Drinking and Smoking Behaviors in Young Adults. Journal of Studies on Alcohol and Drugs, 73(5): 829-833.

Ruisoto P., Cacho R., López-Goñi J.J., Vaca S., Jiménez M. (2016). Prevalence and profile of alcohol consumption among university students in Ecuador. Gaceta Sanitaria, 30(5): 370-374.

Simonato L., Agudo A., Ahrens W., Benhamou E., Benhamou S., Boffetta P., Brennan P., Darby S.C., Forastiere F., Fortes C., Gaborieau V., Gerken M., Gonzales C.A., Jockel K.H., Kreuzer M., Merletti F., Nyberq F., Pershagen G., Pohlabeln H., Rosch F., Whitley E., Wichmann H.E., Zambon P. (2001). Lung cancer and cigarette smoking in Europe: an update of risk estimates and an assessment of inter-country heterogeneity. International Journal of Cancer, 91(6): 876-887.

Sotos J.R., Loper G.A., Parraga M.I., Campos R.M., Simarro H.M.J., Lopez-Torres H.J. (2015). Prevalence of hazardous drinking among nursing students. Journal of Advanced Nursing, 71(3): 581-590.

Suzuki K., Ohida T., Yokoyama E., Kaneita Y., Takemura S. (2005). Smoking among Japanese nursing students: nationwide survey. Journal of Advanced Nursing, 49(3): 268-275.

Watson H., Whyte R., Schartau E. \& Jamieson E. (2006). Survey of student nurses and midwives: smoking and alcohol use. British Journal of Nursing, 15(22): 1212-1216.

Wiencke J.K., Kelsey K.T. (2002). Teen smoking, field cancerization, and a "critical period" hypothesis for lung cancer susceptibility. Environmental Health Perspectectives, 110(6): 555-558.

Wolf L., Stidham A.W., Ross R. (2015). Predictors of stress and coping strategies of US accelerated vs. generic baccalaureate nursing students: an embedded mixed methods study. Nurse Education Today, 35(1): 201-205.

World Health Organization (2002). The World Health Report 2002. Reducing risks, promoting healthy life. Geneva: World Health Organization.

Si ringrazia il Laboratorio Farmaceutico C.T. per il supporto alla realizzazione della newsletter.

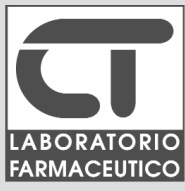

